\title{
Different efficacies of neutralizing antibodies and antiviral drugs on SARS- CoV-2 Omicron subvariants, BA.1 and BA.2
}

Hirofumi Ohashi ${ }^{1 \dagger}$, Takayuki Hishiki ${ }^{1 \dagger}$, Daisuke Akazawa ${ }^{1 \dagger}$, Kwang Su Kim ${ }^{2 \dagger}$, Joohyeon Woo ${ }^{2 \dagger}$, Kaho Shionoya ${ }^{3,4}$, Kana Tsuchimoto ${ }^{1}$, Shoya Iwanami ${ }^{2}$, Saya Moriyama $^{1}$, Hitomi Kinoshita ${ }^{5}$, Souichi Yamada $^{5}$, Yudai Kuroda ${ }^{6}$, Tsukasa Yamamoto $^{6}$, Noriko Kishida7, Shinji Watanabe ${ }^{7}$, Hideki Hasegawa7 ${ }^{7}$ Hideki Ebihara ${ }^{5}$, Tadaki Suzuki ${ }^{8}$, Ken Maeda ${ }^{6}$, Shuetsu Fukushi ${ }^{5}$, Yoshimasa Takahashi ${ }^{1}$, Shingo Iwami $2,9,10,11,12,13 *$, Koichi Watashi1,3,4*

${ }^{1}$ Research Center for Drug and Vaccine Development, National Institute of Infectious Diseases, Tokyo 162-8640, Japan; ${ }^{2}$ Interdisciplinary Biology Laboratory (iBLab), Division of Biological Science, Graduate School of Science, Nagoya University, Nagoya, Japan; ${ }^{3}$ Department of Virology II, National Institute of Infectious Diseases, Tokyo 162-8640, Japan; ${ }^{4}$ Department of Applied Biological Science, Tokyo University of Science, Noda 278-8510, Japan; ${ }^{5}$ Department of Virology I, National Institute of Infectious Diseases, Tokyo 162-8640, Japan; ${ }^{6}$ Department of Veterinary Science, National Institute of Infectious Diseases, Tokyo 162-8640, Japan; ${ }^{7}$ Center for Influenza and Respiratory Virus Research, National Institute of Infectious Diseases; Tokyo 208-0011, Japan; ${ }^{8}$ Department of Pathology, National Institute of Infectious Diseases; Tokyo, 162-8640, Japan; ${ }^{9}$ Institute of Mathematics for Industry, Kyushu University, Fukuoka, Japan; ${ }^{10}$ Institute for the Advanced Study of Human Biology (ASHBi), Kyoto University, Kyoto, Japan; ${ }^{11}$ Interdisciplinary Theoretical and Mathematical Sciences Program (iTHEMS), RIKEN, Saitama, Japan; ${ }^{12}$ NEXT-Ganken Program, Japanese Foundation for Cancer Research (JFCR), Tokyo, Japan; ${ }^{13}$ Science Groove Inc., Fukuoka, Japan 8100041.

†These authors equally contributed to this work.

${ }^{*}$ Corresponding author:

Shingo Iwami: iwami.iblab@bio.nagoya-u.ac.jp

Koichi Watashi: kwatashi@niid.go.jp (Lead contact) 


\section{Summary}

The severe acute respiratory syndrome coronavirus 2 (SARS-CoV-2) Omicron subvariant BA.2 has spread in many countries, replacing the earlier Omicron subvariant BA.1 and other variants. Here, using a cell culture infection assay, we quantified the intrinsic sensitivity of BA. 2 and BA.1 compared with other variants of concern, Alpha, Gamma, and Delta, to five approved-neutralizing antibodies and antiviral drugs. Our assay revealed the diverse sensitivities of these variants to antibodies, including the loss of response of both BA.1 and BA.2 to casirivimab and of BA.1 to imdevimab. In contrast, EIDD-1931 and nirmatrelvir showed a more conserved activities to these variants. The viral response profile combined with mathematical analysis estimated differences in antiviral effects among variants in the clinical concentrations. These analyses provide essential evidence that gives insight into variant emergence's impact on choosing optimal drug treatment.

\section{Keywords}

SARS-CoV-2, Omicron, BA.2, BA.1, casirivimab, imdevimab, sotrovimab, molnupiravir, nirmatrelvir, antiviral 


\section{Introduction}

The severe acute respiratory syndrome coronavirus 2 (SARS-CoV-2) Omicron variant (lineage B.1.1.529) has rapidly spread worldwide and become the most prevalent SARS-CoV-2 in many countries (Elliott et al., 2022; Viana et al., 2022). Of the identified Omicron subvariants, the subvariant BA.1 was dominantly prevalent in the early days after Omicron emerged from November 2021. However, the replacement of BA.1 with another subvariant, BA.2, has grown in the countries, including Denmark, UK, and South Africa, alerting a higher transmission of this new subvariant worldwide that can prolong the current wave of COVID-19 (USKH, 2022). The BA.1 and BA.2 have more than 30 shared amino acid substitutions from the Wuhan strain, especially with approximately 20 shared mutations in the Spike protein. They also have some unique mutations (Figure 1). For example, the S1 69-70 deletion as a hallmark of BA.1, associated with S-gene target failure in PCR tests, is unconserved in BA.2 (WHO, 2021; Majumdar and Sarkar, 2021). BA.2 also has four unique substitutions (S371F, T376A, D405N, and R408S) compared with BA.1, with lacking three mutations (S371L, G446S, and G496S) in the receptorbinding domain of the $\mathrm{S} 1$, which is involved in vaccine and antibody responses (Majumdar and Sarkar, 2021). Such unique mutation patterns in BA.1 and BA.2 possibly affect their sensitivities to approved drugs/antibodies against COVID-19. Therefore, we quantified such drug/antibody responses of BA1 and BA. 2 compared to other variants of concern (Alpha, Gamma, Delta) and a Wuhan strain in cell culture infection assays. Furthermore, most reports have so far evaluated only $50 \%$ (or $90 \%$ ) inhibitory concentrations to quantify the drug activity. Yet, these concentrations are pharmacologically not the sole factor that determines antiviral efficacy. Thus, we also estimated the slopes of dose-response sigmoid curves to quantitatively discuss their drug effects at clinical drug concentrations (Koizumi et al., 2017; Shen et al., 2008).

\section{Results and Discussion}

Dose response curves of approved-antibodies/drugs on SARS-CoV-2 variants.

We evaluated the intrinsic sensitivity of SARS-CoV-2 variants (Wuhan, Alpha, Gamma, Delta, Omicron-BA.1, and Omicron-BA.2) to the approved antibodies/drugs [casirivimab, imdevimab, S309 (the prototype antibody of sotrovimab), EIDD-1931 (the active form of molnupiravir), and nirmatrelvir]. Each SARS-CoV-2 strain was inoculated and cultivated in VeroE6/TMPRSS2 cells upon treatment with varying concentrations of antibodies/drugs (up to $4-10 \mu \mathrm{M}$ or $\mu \mathrm{g} / \mathrm{mL}$ ) to measure viral RNA 
in the culture supernatant, as well as cell viability at $24 \mathrm{~h}$ postinoculation (Matsuyama et al., 2020). Figure 2 shows the dose-response curve of each variant against tested antibodies/drugs (Figure 2). No cytotoxicity induced by antibody/drug was observed in all tested concentrations (Figure S1). Overall, inhibition potency of the three tested antibodies, casirivimab, imdevimab, and S309 to Omicron subvariants BA.1 and BA.2, were severely impaired, in contrast to their outstanding activities against the Wuhan strain and Alpha, Gamma, and Delta variants (Figure 2A-C). Casirivimab did not show any antiviral activity to BA.1 and BA.2 up to $10 \mu \mathrm{g} / \mathrm{ml}$ (Figure 2A). Also, imdevimab lost its activity to BA.1, but retained a minor antiviral activity to reduce BA.2 infections (Figure 2B). S309's antiviral activity to BA.1 was more modest than that of other variants, and that to BA.2 was even weaker (Figure 2C). These tendencies of the $\mathrm{IC}_{50}$ shifts between BA.1 and other variants (Table. 1) are overall consistent with the previous reports (Cameroni et al., 2021; Cao et al., 2021; Liu et al., 2021; Planas et al., 2021). Additionally, our dose-response curves clearly show the impaired potency of all three antibodies against BA.2. As a possible mechanistic explanation, a class 2 antibody, casirivimab, completely lost its antiviral activity to both BA.1 and BA.2, probably because of the mutations at K417N, S477N, T478K, E484A, Q493R, Q498R, and N501Y (Figure 1, black), contained in the reported epitope footprints of casirivimab (VanBlargan et al., 2022). The class 3 imdevimab showed the reduced activity to BA.2 compared with the Wuhan and other variants, which can be explained by the N440K, Q498R, and N501Y mutations (Figure 1, black) within the imdevimab epitope amino acids, and were inactive to BA.1, carrying a further mutation at G446S (Figure 1, blue) within the imdevimab epitope. Another class 3 S309 also showed a reduced antiviral activity against BA.1 and BA.2, containing G339D and N440K substitutions from the Wuhan strain (Figure 1, black) within the S309 epitope footprint. In contrast to antibodies, a polymerase inhibitor, EIDD1931, and a main protease inhibitor, nirmatrelvir, dose-dependently reduced the viral RNA of all variants and showed no resistance (Figure 2D, E), consistent with the previous reports showing no remarkable $\mathrm{IC}_{50}$ differences among BA.1 and other variants (Li et al., 2022; Takashita et al., 2022; Vangeel et al., 2022).

\section{Mathematical analysis for the antibody/drug efficacy on SARS-CoV-2 variants.}

Based on the dose-response curves, we quantified the concentrations that achieved $50 \%$ and $90 \%$ of the maximal effect ( $\mathrm{IC}_{50}, \mathrm{IC}_{90}$ ). Additionally, we estimated the Hill coefficient (m) (Koizumi et al., 2017; Shen et al., 2008), showing the steepness of the sigmoid curve, given by the equation for a fraction of infection 
events unaffected by drugs $\left(\mathrm{f}_{\mathrm{u}}\right)$,

$$
\mathrm{f}_{\mathrm{u}}=1 /\left\{1+\left(\mathrm{D} / \mathrm{IC}_{50}\right)^{\mathrm{m}}\right\}
$$

Although $\mathrm{IC}_{50}$ (or $\mathrm{IC}_{90}$ ) is frequently used to evaluate the "potency" of drugs, the "efficacy" of drugs is determined by both $\mathrm{m}$ and $\mathrm{IC}_{50}$ : The inhibition of viral propagation ("drug efficacy") at any given drug concentration (D) can be expressed as the instantaneously inhibitory potential (IIP) (Koizumi et al., 2017; Shen et al., 2008).

$$
\mathrm{IIP}=\log \left[1+\left(\mathrm{D} / \mathrm{IC}_{50}\right)^{\mathrm{m}}\right]
$$

Here IIP indicates the log of viral reduction. Since antiviral drugs are usually at far higher concentrations than IC $_{50}$ in clinical settings, the high steepness of the sigmoid curve (high $\mathrm{m}$ ) achieves a much higher IIP than those having low steepness (low $\mathrm{m}$ ) even if $\mathrm{IC}_{50}$ is the same. Hence, since sequence substitutions in the drug target [Spike, main protease (NSP5), or polymerase (NSP12)] can change IC $_{50}$ and $\mathrm{m}$, we estimated these values based on the dose-response curve for each variant through the fitting of $f_{u}$ with nonlinear least squared regression (Table 1). To evaluate the antiviral effect in clinical drug concentrations, we calculated IIP at maximum drug concentrations $\left[\mathrm{IIP}\left(\mathrm{C}_{\max }\right)\right]$ for each antibody/drug against each variant (Table 1 and Table S1) based on available pharmacokinetics in patients. Although casirivimab conserved $\mathrm{IC}_{50}$ and $\mathrm{m}$ (within two folds) among the Wuhan, Alpha, Gamma, and Delta strains, with profound effects to reduce viral propagation at $\mathrm{C}_{\max }$ (3.67-7.00 log), its activity was lost to BA.1 and BA.2. While imdevimab also had a conserved antiviral effect at $C_{\max }$ (4.52-7.97 log) to the variants other than Omicron, its effect was lost on BA.1, and was retained moderately on BA.2 (2.17 log). S309's effect at $C_{\max }$ on these variants other than BA.2 was high ( $>3 \log$ ), but that on BA. 2 was estimated to be lower than others $(1.15 \mathrm{log})$. In contrast, the $\mathrm{IC}_{50}$ and $\mathrm{m}$ for EIDD-1931 against each variant were less diverse (within two folds) to conserve strong antiviral effects at $\mathrm{C}_{\max }$ (1.97-3.53 log). Nirmatrelvir had an even more conserved $\mathrm{IC}_{50}$ and $\mathrm{m}$, with similar IIP at $\mathrm{C}_{\max }(0.753-0.986)$. These analyses suggest that while the three approved-antibodies were less active to BA.1 and BA.2, EIDD-1931 and nirmatrelvir had conserved antiviral effects on variants. Our analysis suggests that the emergence of SARS-CoV-2 variants narrowed the options for efficient antibody treatments.

\section{Limitations of the study}

This study was limited to cell culture infection assays, in which cell types and other conditions reflected drug sensitivities. However, viral targeting agents examined in this study (casirivimab, imdevimab, and S309 target Spike, EIDD-1931 
target polymerase, and nirmatrelvir target main protease) were much less governed through cellular backgrounds, compared to host-targeting antivirals, and instead are more affected by viral factors such as sequence changes in the viral genome. Our assay at least compared the intrinsic antibody/drug sensitivity of SARS-CoV-2 variants side by side, helpful in discussing the impact of sequence substitutions on antibody/drug activities. Additionally, the analyses of animal and patient infections under treatment were further desired to understand drug efficacy. Yet, given the Omicron BA.2 wave's urgency and the need for the scientific evidence to better combat this infectious disease, our data significantly present the potential diversity of drug/antibody efficacies among SARS-CoV-2 variants.

\section{Acknowledgments}

VeroE6/TMPRSS2 cells were kindly provided by Dr. Makoto Takeda at Department of Virology III, National Institute of Infectious Diseases. This study was supported by an AMED grant JP20fk0108411 (to K.W.), Moonshot R\&D Grant JPMJMS2021 (to S.I.), JPMJMS2025 (to S.I.), and JST MIRAI (to S.I. and K.W.)

\section{Author contributions}

H.O., T.H., D.A., K.S., K.T., and K.W. performed experiments. K.S.K., J.W., S.Iwanami, and S.Iwami performed mathematical analysis. S.M., H.K., S.Y., Y.K., T.Y., N.K., S.W., H.H., H.E., T.S., K.M., S.F., and Y.T. contributed materials. S.Iwami and K.W. prepared manuscripts. K.W. supervised project.

\section{Competing interests}

The authors declare no competing interests.

\section{Methods}

\section{Cell culture infection assay}

VeroE6/TMPRSS2 cells, a VeroE6 cell clone overexpressing the transmembrane protease, serine 2 (TMPRSS2), were cultured in Dulbecco's modified Eagle's medium (Life Technologies) supplemented with 10\% fetal bovine serum (FBS) (Sigma), 100 units/mL penicillin, $100 \mu \mathrm{g} / \mathrm{mL}$ streptomycin, $10 \mathrm{mM}$ HEPES (pH 7.4), 
and $1 \mathrm{mg} / \mathrm{mL}$ G418 (Nacalai) at $37^{\circ} \mathrm{C}$ in 5\% CO2 (Ohashi et al., 2021).

SARS-CoV-2 was investigated in a biosafety level 3 (BSL3) room. Through viral inoculation, we used WK-521 (Wuhan strain, EPI_ISL_408667), QK002 (Alpha, EPI_ISL_768526), TY7-501 (Gamma, EPI_ISL_833366), TY11-927 (Delta, EPI_ISL_2158617), TY38-873 (Omicron BA.1, EPI_ISL_7418017), and TY40-385 (Omicron BA.2, EPI_ISL_9595859) strains, isolated from COVID-19 patients and registered in GISAID. Viral infectious titers were measured by inoculating VeroE6/TMPRSS2 cells with a 10-fold serial dilution of the virus followed by measuring cytopathology to calculate $\mathrm{TCID}_{50} / \mathrm{ml}$ (Ohashi et al., 2021). Infection assay was conducted by inoculating VeroE6/TMPRSS2 cells with each SARS-CoV-2 strain at an MOI of 0.003 for $1 \mathrm{~h}$, followed by washing out free viruses and culturing cells with fresh medium. A medium with $2 \%$ FBS without G418 was used during the infection assay. Antibodies and antiviral drugs were treated for $1 \mathrm{~h}$ during virus inoculation and $24 \mathrm{~h}$ after inoculation. At $24 \mathrm{~h}$ postinfection, the culture supernatant was recovered to isolate RNA using MagMax Viral/Pathogen Nucleic Acid Isolation kit (Thermo Fisher Scientific) and quantify SARS-CoV-2 RNA by real time RT-PCR with a one-step qRT-PCR kit (THUNDERBIRD Probe One-step qRT-PCR kit, TOYOBO) using 5'- ACAGGTACGTTAATAGTTAATAGCGT-3', 5'ATATTGCAGCAGTACGCACACA-3', and 5'-FAMACACTAGCCATCCTTACTGCGCTTCG-TAMRA-3'. Simultaneously, cell viability was quantified by fixing the cells with $4 \%$ paraformaldehyde and staining with DAPI to count the number of cells using a high content imaging system ImageXpress (Molecular Devices).

\section{References}

1. Cameroni, E., Bowen, J.E., Rosen, L.E., Saliba, C., Zepeda, S.K., Culap, K., Pinto, D., VanBlargan, L.A., De Marco, A., di Iulio, J., et al. (2021). Broadly neutralizing antibodies overcome SARS-CoV-2 Omicron antigenic shift. Nature. doi: 10.1038/s41586-021-04386-2

2. Cao, Y., Wang, J., Jian, F., Xiao, T., Song, W., Yisimayi, A., Huang, W., Li, Q., Wang, P., An, R., et al. (2021). Omicron escapes the majority of existing SARS-CoV-2 neutralizing antibodies. Nature. doi: 10.1038/s41586-021-04385-3

3. Elliott, P., Bodinier, B., Eales, O., Wang, H., Haw, D., Elliott, J., Whitaker, M., Jonnerby, J., Tang, D., Walters, C.E., et al. (2022). Rapid increase in Omicron infections in England during December 2021: REACT-1 study. Science, eabn8347. doi: 


\section{$10.1126 /$ science.abn8347}

4. Koizumi, Y., Ohashi, H., Nakajima, S., Tanaka, Y., Wakita, T., Perelson, A.S., Iwami, S., and Watashi, K. (2017). Quantifying antiviral activity optimizes drug combinations against hepatitis C virus infection. Proc Natl Acad Sci U S A 114, 1922-1927. doi: 10.1073/pnas.1610197114

5. Li, P., Wang, Y., Lavrijsen, M., Lamers, M.M., de Vries, A.C., Rottier, R.J., Bruno, M.J., Peppelenbosch, M.P., Haagmans, B.L., and Pan, Q. (2022). SARS-CoV-2 Omicron variant is highly sensitive to molnupiravir, nirmatrelvir, and the combination. Cell Res. doi: 10.1038/s41422-022-00618-w

6. Liu, L., Iketani, S., Guo, Y., Chan, J.F., Wang, M., Liu, L., Luo, Y., Chu, H., Huang, Y., Nair, M.S., et al. (2021). Striking antibody evasion manifested by the Omicron variant of SARS-CoV-2. Nature. doi: 10.1038/s41586-021-04388-0

7. Majumdar, S., and Sarkar, R. (2021). Mutational and phylogenetic analyses of the two lineages of the Omicron variant. J Med Virol. doi: 10.1002/jmv.27558

8. Matsuyama, S., Nao, N., Shirato, K., Kawase, M., Saito, S., Takayama, I., Nagata, N., Sekizuka, T., Katoh, H., Kato, F., et al. (2020). Enhanced isolation of SARS-CoV-2 by TMPRSS2-expressing cells. Proc Natl Acad Sci U S A 117, 7001-7003. doi: 10.1073/pnas.2002589117

9. Ohashi, H., Watashi, K., Saso, W., Shionoya, K., Iwanami, S., Hirokawa, T., Shirai, T., Kanaya, S., Ito, Y., Kim, K.S., et al. (2021). Potential anti-COVID-19 agents, cepharanthine and nelfinavir, and their usage for combination treatment. iScience 24, 102367. doi: 10.1016/j.isci.2021.102367

10. Planas, D., Saunders, N., Maes, P., Guivel-Benhassine, F., Planchais, C., Buchrieser, J., Bolland, W.H., Porrot, F., Staropoli, I., Lemoine, F., et al. (2021). Considerable escape of SARS-CoV-2 Omicron to antibody neutralization. Nature. doi: 10.1038/s41586-021-04389-z

11. Shen, L., Peterson, S., Sedaghat, A.R., McMahon, M.A., Callender, M., Zhang, H., Zhou, Y., Pitt, E., Anderson, K.S., Acosta, E.P., et al. (2008). Dose-response curve slope sets class-specific limits on inhibitory potential of anti-HIV drugs. Nat Med 14, 762-766. doi: $10.1038 / \mathrm{nm} 1777$

12. Takashita, E., Kinoshita, N., Yamayoshi, S., Sakai-Tagawa, Y., Fujisaki, S., Ito, M., Iwatsuki-Horimoto, K., Chiba, S., Halfmann, P., Nagai, H., et al. (2022). Efficacy of Antibodies and Antiviral Drugs against Covid-19 Omicron Variant. N Engl J Med. doi: 10.1056/NEJMc2119407

13. UK Health Security Agency (UKSH). (2022). SARS-CoV-2 variants of concern and variants under investigation in England. Technical briefing 35. 28 January 2022 
14. VanBlargan, L.A., Errico, J.M., Halfmann, P.J., Zost, S.J., Crowe, J.E., Jr., Purcell, L.A., Kawaoka, Y., Corti, D., Fremont, D.H., and Diamond, M.S. (2022). An infectious SARS-CoV-2 B.1.1.529 Omicron virus escapes neutralization by therapeutic monoclonal antibodies. Nat Med. doi: 10.1038/s41591-021-01678-y

15. Vangeel, L., Chiu, W., De Jonghe, S., Maes, P., Slechten, B., Raymenants, J., Andre, E., Leyssen, P., Neyts, J., and Jochmans, D. (2022). Remdesivir, Molnupiravir and Nirmatrelvir remain active against SARS-CoV-2 Omicron and other variants of concern. Antiviral Res 198, 105252. doi: 10.1016/j.antiviral.2022.105252

16. Viana, R., Moyo, S., Amoako, D.G., Tegally, H., Scheepers, C., Althaus, C.L., Anyaneji, U.J., Bester, P.A., Boni, M.F., Chand, M., et al. (2022). Rapid epidemic expansion of the SARS-CoV-2 Omicron variant in southern Africa. Nature. doi: 10.1038/s41586-022-04411-y

17. World Health Organization (WHO). (2021). Classification of Omicron (B.1.1.529): SARS-CoV-2 Variant of Concern 


\section{Figure legends}

Figure 1. Schematic representations for amino acid substitutions within the B.1.1.7, P.1, B.1.617.2, BA.1, and BA.2 lineage in NSP5, NSP12, and Spike proteins. Upper boxes show coding regions for NSP5 (the target of nirmatrelvir), NSP12 (the target of EIDD-1931), and Spike (the target of imdevimab, casirivimab, and S309) in the SARS-CoV-2 genome RNA. Mutated amino acids from the Wuhan strain in B.1.1.7 (Alpha, orange), P.1 (Gamma, green), B.1.617.2 (Delta, yellow), BA.1 (Omicron), and BA.2 (Omicron) are shown. Shared BA.1 and BA.2 mutations are indicated in black, and those unique to BA.1 and BA.2 are shown in blue and red, respectively.

Figure 2. Dose-response curves for each SARS-CoV-2 variant propagation upon antibody or drug treatment. Relative SARS-CoV-2 RNAs were plotted in log-scale against the concentrations of approved antibodies/drugs [A: casirivimab, B: imdevimab, C: S309 (a parent antibody of sotrovimab), D: EIDD-1931 (the active form of molnupiravir), and E: nirmatrelvir]. Gray: Wuhan strain, orange: Alpha, green: Gamma, yellow: Delta, blue: Omicron-BA.1, and red: Omicron-BA.2. Data are presented as mean \pm SD across the three replicate experiments. Relative values are shown as percentages of viral RNA in culture supernatants to the control wells incubated without antibodies/drugs. Values less than $0.1 \%$ are shown as $0.1 \%$ in these graphs. 
Table 1. Estimated parameters for the antiviral effect of drugs

\begin{tabular}{lllcc}
\hline & $\boldsymbol{I}_{\mathbf{5 0}}$ & $\boldsymbol{I} \boldsymbol{C}_{\mathbf{9 0}}$ & $\boldsymbol{m}$ & $\boldsymbol{I I P}\left(\boldsymbol{C}_{\boldsymbol{m a x}}\right)$ \\
\hline casirivimab & $(\mu \mathrm{g} / \mathrm{ml})$ & $(\mu \mathrm{g} / \mathrm{ml})$ & & \\
\hline WK-521 & 0.0139 & 0.0713 & 1.3444 & 5.5662 \\
QK002 (Alpha) & 0.0136 & 0.0505 & 1.6747 & 6.9496 \\
TY7-501 (Gamma) & 0.0140 & 0.1666 & 0.8872 & 3.6706 \\
TY11-927 (Delta) & 0.0217 & 0.0749 & 1.7743 & 7.0029 \\
TY38-873 (Omicron. BA.1) & $>10$ & - & - & - \\
TY40-385 (Omicron. BA.2) & $>10$ & - & - & - \\
\hline imdevimab & $(\mu \mathrm{g} / \mathrm{ml})$ & $(\mu \mathrm{g} / \mathrm{ml})$ & & \\
\hline WK-521 & 0.0125 & 0.0963 & 1.0763 & 4.5202 \\
QK002 (Alpha) & 0.0227 & 0.0813 & 1.7221 & 6.7862 \\
TY7-501 (Gamma) & 0.0082 & 0.0416 & 1.3529 & 5.9296 \\
TY11-927 (Delta) & 0.0290 & 0.0834 & 2.0809 & 7.9787 \\
TY38-873 (Omicron. BA.1) & $>10$ & - & - & - \\
TY40-385 (Omicron. BA.2) & 1.2525 & 11.6931 & 0.9836 & 2.1658 \\
\hline S309 & $(\mu \mathrm{g} / \mathrm{ml})$ & $(\mu \mathrm{g} / \mathrm{ml})$ & & \\
\hline WK-521 & 0.1587 & 0.7583 & 1.4048 & 4.0316 \\
QK002 (Alpha) & 0.0552 & 0.5449 & 0.9596 & 3.1943 \\
TY7-501 (Gamma) & 0.0384 & 0.1498 & 1.6143 & 5.6276 \\
TY11-927 (Delta) & 0.0870 & 0.2407 & 2.1589 & 6.7593 \\
TY38-873 (Omicron. BA.1) & 0.9579 & 2.6822 & 2.1348 & 4.4598 \\
TY40-385 (Omicron. BA.2) & 1.3579 & 62.2520 & 0.5744 & 1.1452 \\
\hline EIDD-1931 & $(\mu \mathrm{M})$ & $(\mu \mathrm{M})$ & & \\
\hline WK-521 & 0.2270 & 0.7385 & 1.8626 & 2.9789 \\
QK002 (Alpha) & 0.3435 & 1.0099 & 2.0375 & 2.8922 \\
TY7-501 (Gamma) & 0.2432 & 0.6531 & 2.2241 & 3.4901 \\
TY11-927 (Delta) & 0.2828 & 1.4743 & 1.3307 & 2.0052 \\
TY38-873 (Omicron. BA.1) & 0.3407 & 1.6655 & 1.3846 & 1.9746 \\
TY40-385 (Omicron. BA.2) & 0.4614 & 1.0315 & 2.7310 & 3.5260 \\
\hline nirmatrelvir & $(\mu \mathrm{M})$ & $(\mu \mathrm{M})$ & & \\
\hline WK-521 & 1.8787 & 4.8869 & 2.5227 & 0.9858 \\
QK002 (Alpha) & 1.7795 & 6.5284 & 1.6904 & 0.7530 \\
TY7-501 (Gamma) & 1.6458 & 4.8727 & 2.0243 & 0.9244 \\
TY11-927 (Delta) & 1.7959 & 5.1161 & 2.0988 & 0.8828 \\
TY38-873 (Omicron. BA.1) & 1.8522 & 4.7245 & 2.3465 & 0.9403 \\
TY40-385 (Omicron. BA.2) & 1.9402 & 5.1784 & 2.2393 & 0.8653 \\
\hline & & & & \\
\hline
\end{tabular}

- : The value cannot be estimated because of the low antiviral activity 
Fig. 1

\section{SARS-CoV-2}

B.1.1.7 (Alpha)

$$
\text { P.1 (Gamma) }
$$

B.1.617.2 (Delta)

BA.1 (Omicron)

BA.2 (Omicron)
P132Y

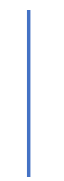

\section{NSP5}

NSP12

P323L

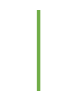

P323L

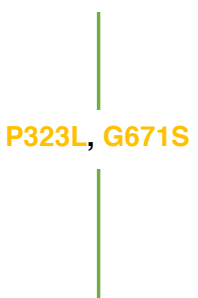

P323L

P132H
P323L

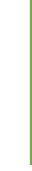

\section{Spike}

69-70del, 144del, N501Y, A570D, D614G, P681H, T716I, S982A, D1118H

L18F, T20N, P26S, D138Y, G181V, R190S, K417T, E484K, N501Y, D614G, H655Y, T1027I, V1176F

T19R, G142D, E156G, 157-158del, L452R, T478K, D614G, P681R, D950N

A67V, 69-70del, T95I, G142D, 143-145del, 211del, L212I, ins214EPE, G339D, S371L, S373P, S375F, K417N, N440K, G446S, S477N, T478K, E484A, Q493R, G496S, Q498R, N501Y, Y505H, T547K, D614G, H655Y, N679K, P681H, N764K, D796Y, N856K, Q954H, N969K, L981F

T19I, 24-26del, A27S, G142D, V213G, G339D, S371F, S373P, S375F, T376A, D405N, R408S, K417N, N440K, S477N, T478K, E484A, Q493R, Q498R, N501Y, Y505H, D614G, H655Y, N679K, P681H, N764K, D796Y, Q954H, N969K 
Fig. 2
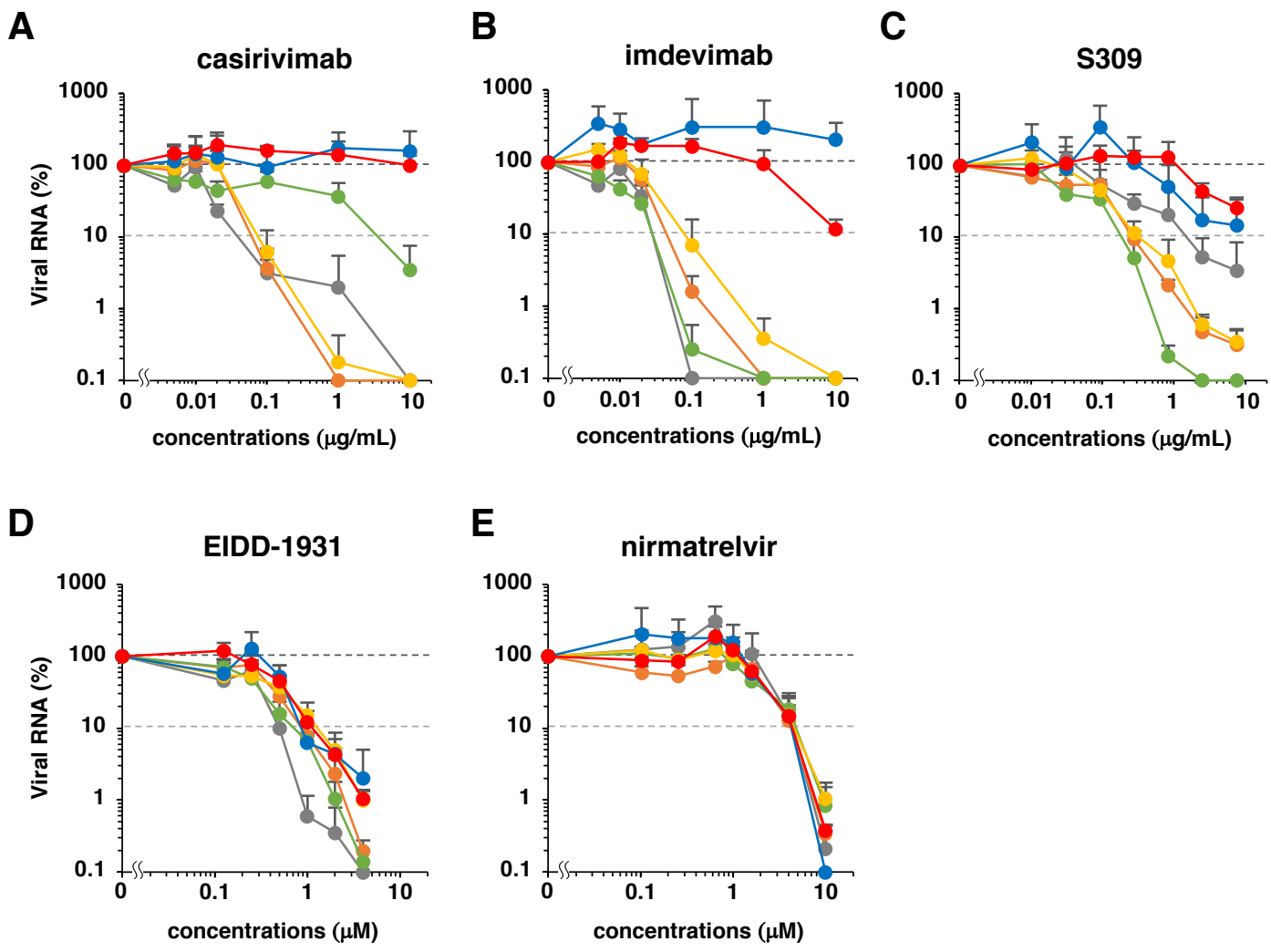

$\multimap$ Wuhan $\multimap$ Alpha $\multimap$ Gamma $\multimap$ Delta $\multimap$ Omicron-BA.1 $\multimap$ Omicron-BA.2 